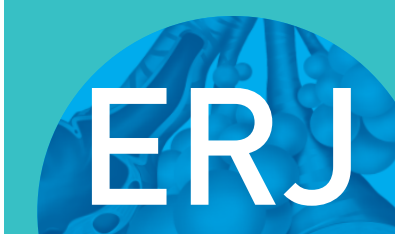

open research
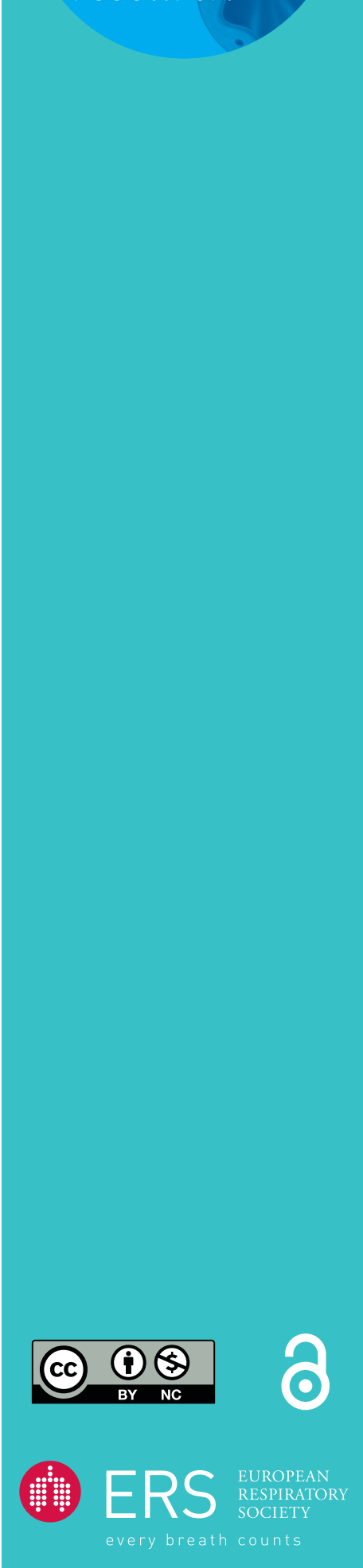

\section{Oxygen therapy in COPD and interstitial lung disease: navigating the knowns and unknowns}

\author{
Yet H. Khor (1) ${ }^{1,2,3,4}$, Elisabetta A. Renzoni ${ }^{5}$, Dina Visca ${ }^{6,7}$, \\ Christine F. McDonald ${ }^{1,2,4}$ and Nicole S. L. Goh ${ }^{1,2,3}$
}

Affiliations: ${ }^{1}$ Dept of Respiratory and Sleep Medicine, Austin Health, Heidelberg, Australia. ${ }^{2}$ Institute for Breathing and Sleep, Heidelberg, Australia. ${ }^{3}$ Dept of Respiratory Medicine, Alfred Health, Melbourne, Australia. ${ }^{4}$ School of Medicine, University of Melbourne, Melbourne, Australia. ${ }^{5}$ Interstitial Lung Disease Unit, Royal Brompton Hospital, Imperial College London, London, UK. ${ }^{6}$ Division of Pulmonary Rehabilitation, Istituti Clinici Scientifici Maugeri, IRCCS, Tradate, Italy. ${ }^{7}$ Dept of Medicine and Surgery, Respiratory Diseases, University of Insubria, Varese-Como, Italy.

Correspondence: Yet H. Khor, Dept of Respiratory and Sleep Medicine, Austin Health, 145 Studley Road, Heidelberg, 3084 VIC, Australia. E-mail: yethong.khordaustin.org.au

ABSTRACT Domiciliary oxygen therapy is often prescribed for patients with hypoxaemia due to advanced lung disease, most commonly chronic obstructive pulmonary disease (COPD) and interstitial lung disease (ILD). Long-term oxygen therapy (LTOT) trials conducted in patients with COPD in the 1980s remain the basis for clinical decisions and guideline recommendations regarding LTOT for patients with non-COPD conditions as there is a lack of high-quality evidence concerning its use in the nonCOPD population. There is also a lack of evidence for the use of ambulatory and nocturnal oxygen therapy in patients with isolated exertional and nocturnal hypoxaemia. These deficiencies pose significant challenges in patient care, with consequent discrepancies in guideline recommendations and clinical approaches. In recent years, new studies have been and are currently being conducted to fill the gaps in our understanding and use of domiciliary oxygen therapy for other indications, including ILD. This article provides a comparison of the epidemiology and significance of hypoxaemia in patients with COPD and ILD, with an up-to-date review of current evidence regarding the role of different types of domiciliary oxygen therapy in these conditions.

@ERSpublications

Despite the significance of hypoxaemia in patients with chronic lung diseases, an up-to-date review shows current evidence for clinical use of domiciliary oxygen therapy remains limited http://bit.ly/33aW31n

Cite this article as: Khor YH, Renzoni EA, Visca D, et al. Oxygen therapy in COPD and interstitial lung disease: navigating the knowns and unknowns. ERJ Open Res 2019; 5: 00118-2019 [https:// doi.org/10.1183/23120541.00118-2019].

Received: 14 May 2019 | Accepted after revision: 29 July 2019

Copyright $\odot$ ERS 2019. This article is open access and distributed under the terms of the Creative Commons Attribution Non-Commercial Licence 4.0. 


\section{Introduction}

The concept of oxygen as a therapeutic agent was first recognised in the late 18th century by Thomas Beddoes, the father of respiratory therapy [1]. It was not until the early 20th century that clinical values of oxygen were better appreciated with the discoveries of its physiological effects. Domiciliary oxygen therapy was then pioneered in the 1950 s by Cotes in the UK and Barach in the USA, with the development of portable delivery systems [2,3]. Today, domiciliary oxygen therapy is commonly prescribed for patients with chronic hypoxaemia. The use of domiciliary oxygen therapy has been increasing worldwide in recent years, accounting for substantial health expenditure. The national incidence of domiciliary oxygen therapy in Sweden increased from 3.9 to 14.7 per 100000 population between 1987 and 2015 [4]. In Australia, the total annual costs for domiciliary oxygen therapy were estimated to be AUD31 million in 2005, excluding indirect clinical costs associated with serial assessment, patient education and support [5]. It is estimated that Medicare expenditures for domiciliary oxygen therapy increased from USD1.7 billion in 1997 to USD2.9 billion in 2008 in the USA [6, 7]. While correcting hypoxaemia may confer physiological benefits, the effects of supplemental oxygen on oxidative stress responses remain unclear [8-11]. More importantly, patients have described a range of physical difficulties and psychological burden with using domiciliary oxygen therapy [12-14]. Globally, chronic obstructive pulmonary disease (COPD) and interstitial lung disease (ILD) are the two most common indications for domiciliary oxygen therapy [4, 15-17]. Despite both diseases sharing clinical features of dyspnoea and exercise limitations, there are marked differences in their prevalence, pathophysiology and natural history. Herein, we review and contrast the epidemiology of hypoxaemia and current evidence regarding the role of domiciliary oxygen therapy in COPD and ILD.

\section{Epidemiology and significance of hypoxaemia in COPD and ILD}

The prevalence of hypoxaemia among patients with COPD and ILD remains unclear. This is in part due to the lack of standardised definitions and test modalities for describing different types of hypoxaemia, including resting, exertional and nocturnal hypoxaemia. In addition, prevalence rates of hypoxaemia vary depending on the selected study population and disease severity. Nevertheless, current literature suggests that hypoxaemia is a significant feature in both COPD and ILD.

Resting hypoxaemia is a marker of advanced disease stages for chronic lung diseases. It is commonly defined as resting arterial oxygen tension $\left(P_{\mathrm{aO}_{2}}\right) \leqslant 55 \mathrm{mmHg}$ or $56-59 \mathrm{mmHg}$ with evidence of end-organ damage (cor pulmonale, polycythaemia and/or pulmonary hypertension). Longitudinal data from the Swedish National Register of COPD reported a resting hypoxaemia prevalence of $1.4 \%$ [18]. Of those with moderate-to-severe COPD, 7\% of patients developed resting hypoxaemia after follow-up for a median of 5 years [19]. There is a paucity of studies evaluating the epidemiology of resting hypoxaemia in ILD in the literature. Recent large randomised controlled trials in idiopathic pulmonary fibrosis, the most common form of ILD, reported that $21-28 \%$ of trial participants were prescribed supplemental oxygen therapy [20, 21$]$. However, these reported rates included any use of domiciliary oxygen therapy, without differentiating resting from isolated exertional hypoxaemia. A recent retrospective study of 400 patients at Australian specialised ILD clinics reported a resting hypoxaemia prevalence of 3.5\% [22]

Definitions of exertional hypoxaemia vary widely in clinical trials, including a nadir arterial oxygen saturation measured by pulse oximetry $\left(S_{\mathrm{pO}_{2}}\right)$ of $\leqslant 88 \%$ [23-26] and a decrease in $S_{\mathrm{pO}_{2}}$ of $\geqslant 4 \%$ with or without a nadir $S_{\mathrm{pO}_{2}}$ of $<90 \%$ [27-31]. Regardless of the definitions used, exertional hypoxaemia is common in patients with COPD and ILD. The prevalence of exertional hypoxaemia ranges from $29 \%$ to $39 \%$ for patients with moderate-to-severe COPD $[25,26]$. In unselected groups of patients with ILD, 49-54\% experience exertional hypoxaemia [22, 32]. Consistent with clinical observation, exertional hypoxaemia is more common in patients with ILD compared with those with COPD. With similar degrees of respiratory physiology impairment, patients with ILD experience more severe exertional hypoxaemia than those with COPD [32]. Nevertheless, exertional hypoxaemia is an important prognostic factor for both COPD [25, 33] and ILD populations [23, 34, 35].

The prevalence of nocturnal hypoxaemia in patients with COPD and ILD varies from $27 \%$ to $70 \%$ [36-39] and $36 \%$ to $57 \%$ [40-43], respectively. In addition to the variations in criteria used to define nocturnal hypoxaemia in these studies, the presence of coexisting sleep disordered breathing may also impact on its prevalence. In both conditions, nocturnal hypoxaemia is only loosely related to daytime resting or exertional hypoxaemia and does not correlate with the degree of lung function impairment [37, 39, 41]. There have been studies that suggest a potential association of nocturnal hypoxaemia with pulmonary hypertension in patients with COPD and ILD [41, 43-45]. However, the presence of sleep disordered breathing, a common comorbidity in patients with both conditions [46-48], was not evaluated in the majority of these studies. The prevalence and prognostic significance of nocturnal hypoxaemia without sleep disordered breathing for both conditions are uncertain. 


\section{Long-term oxygen therapy in COPD and ILD}

Long-term oxygen therapy (LTOT) is typically prescribed to be used for at least $15-18 \mathrm{~h}$ per day for patients with resting hypoxaemia. It is a well-established intervention in patients with COPD and resting hypoxaemia. Therapeutic benefits of LTOT were reported in two pivotal randomised controlled trials published $>30$ years ago: the Nocturnal Oxygen Therapy Trial (NOTT) and the Medical Research Council (MRC) trial [49, 50]. Both trials studied patients with stable severe COPD and significant resting hypoxaemia, as defined previously. In the MRC trial, patients randomised to nocturnal oxygen supplementation for at least $15 \mathrm{~h}$ daily had improved survival compared with the control group at 5-year follow-up $(55 \%$ versus $33 \%$; $\mathrm{p}<0.05)$. The NOTT trial compared the effects of continuous oxygen therapy against nocturnal oxygen therapy for $12 \mathrm{~h}$. Patients who used continuous oxygen therapy consistently had better prognosis at 1-year and 2-year follow-up. These studies provide evidence for survival benefit with LTOT in a subgroup of patients with COPD and highlighted the importance of usage duration for LTOT.

On the contrary, the beneficial effects of LTOT have not been shown in patients with COPD and moderate resting or exertional hypoxaemia. Two trials in the 1990s and 2000s revealed no significant differences in survival between treated and untreated groups [51, 52]. Arguably, both trials were underpowered to detect survival differences. The US-based Long-Term Oxygen Treatment Trial (LOTT), a multicentre unblinded randomised controlled trial of oxygen for patients with COPD, was completed in 2015 [53]. There was no significant difference between treatment and control groups in time to hospital admission or death and other outcomes, including health-related quality of life and symptoms. The LOTT trial encountered many challenges, particularly study recruitment, which resulted in trial design amendments over the course of 5 years. Study eligibility criteria were extended to include patients with moderate exertional desaturation only, in addition to those with moderate resting hypoxaemia. The primary outcome for this trial was also changed from all-cause mortality to a composite primary outcome (time to death or first hospitalisation for any cause). Recruitment challenges faced by the LOTT trial reflect the strong belief in oxygen therapy among healthcare professionals and patients, despite the lack of evidence in this area. Results from the LOTT trial confirmed the lack of efficacy for LTOT in patients with stable COPD and moderate hypoxaemia.

In comparison with the landmark trials of LTOT in COPD, there is a lack of high-quality studies evaluating the efficacy of LTOT in patients with ILD. The only small multicentre controlled study of LTOT conducted in 1988 by Braghiroli and Donner remains unpublished, although some data have been reported in a Cochrane review [54]. In this study, 62 patients with clinically stable ILD and resting hypoxaemia, defined as $P_{\mathrm{aO}}$ between 45 and $60 \mathrm{mmHg}$, were recruited. The majority of patients (79\%) had idiopathic pulmonary fibrosis as defined at the time. There was no significant difference in mortality between the treated and untreated groups, with mortality rates of $91 \%$ for both groups after 3 years. The observed high mortality rates are consistent with findings from retrospective studies of patients with ILD who were on LTOT, showing 50\% of patients died within 1 year of commencing LTOT or home ventilation [55-57]. Despite a lack of evidence, LTOT remains widely accepted for use in patients with ILD and resting hypoxaemia.

\section{Ambulatory oxygen therapy in COPD and ILD}

Ambulatory oxygen therapy (AOT) involves the use of a portable oxygen delivery device during exercise or activities of daily living. This therapy can be prescribed as a stand-alone intervention for patients with isolated exertional hypoxaemia or as a supplement to a stationary oxygen concentrator for LTOT. The range of portable oxygen delivery devices for ambulatory use has expanded from compressed oxygen cylinders in the 1950s [2,3] and liquid oxygen systems in the 1960s [58] to portable oxygen concentrators, first introduced in the 1990s and commercially available since the 2000s [59-61]. Compressed oxygen cylinders are still the most widely used source of AOT globally, with liquid oxygen systems and portable oxygen concentrators being used in some countries.

The therapeutic role of AOT in COPD and ILD remains controversial. The effects of supplemental oxygen during activities in patients with COPD and ILD have been evaluated during in-laboratory exercise tests and in clinical trials. Various exercise tests, including walk tests, treadmill tests and cycle ergometry, have been used for in-laboratory assessments. For both diseases, supplemental oxygen therapy has consistently been shown to improve exercise performance during in-laboratory tests, with some reporting a reduction in exertional dyspnoea [62, 63]. In addition to small study populations, most of these in-laboratory assessment studies had inadequate study design with a lack of blinding and randomisation. Furthermore, it is not possible to translate these findings from in-laboratory assessment studies into daily life given the differences in exercise intensity and functional requirements.

A systematic review of four randomised placebo-controlled trials of AOT in patients with COPD concluded that oxygen improved exertional dyspnoea, without survival benefit or enhanced exercise 
capacity [64]. The review included patients without significant resting hypoxaemia, defined as meeting the criteria for LTOT, with the duration of intervention varying between 2 and 12 weeks. Although the dyspnoea and fatigue domains of health-related quality of life, measured using the Chronic Respiratory Questionnaire, statistically favoured AOT, the improvement did not reach the threshold for clinical significance. The recent LOTT trial showed no beneficial effects in health-related quality of life and functional status with nocturnal and AOT in COPD patients with moderate exertional desaturation, defined as a fall in $S_{\mathrm{pO}_{2}}$ to $<80 \%$ for $\geqslant 5 \mathrm{~min}$ and $<90 \%$ for $\geqslant 10 \mathrm{~s}$ [53]. In a parallel-group double-blinded randomised placebo-controlled trial by MOORE et al. [65], there were no significant differences in dyspnoea, mental wellbeing, health-related quality of life or functional status between intervention and placebo groups. However, only 50 patients included in this study demonstrated exertional desaturation to $\leqslant 88 \%$ during 6-min walk tests at baseline. Randomised trials of patients with COPD on LTOT revealed no beneficial effects from adding AOT to stationary concentrator use [66-68]. It is important to note that there was minimal to little increment in oxygen usage duration with the provision of AOT, regardless of the delivery systems provided for AOT.

The only published randomised prospective trial in patients with ILD, evaluating longer-term effects of AOT, delivered via compressed oxygen cylinders, is the Ambulatory Oxygen in Fibrotic Lung Disease (AmbOx) trial [69]. The AmbOx trial was a multicentre randomised controlled crossover trial in the UK comparing the effects of AOT with no AOT during activities in patients with fibrotic ILD and isolated exertional desaturation. This trial randomly assigned 84 participants to two 2 -week periods of AOT or no AOT, without a washout period in between. AOT significantly improved both the "Breathlessness and activity" and "Chest symptoms" domains of the King's Brief ILD Questionnaire (K-BILD), but not the "Psychological" domain. Although total K-BILD score, a measure of overall health-related quality of life, was improved with AOT (difference 3.7 points; $p<0.0001$ ), the minimal clinically important difference (MCID) of 5 points was not met [70]. However, the MCID was met for the "Breathlessness and activity" domain (difference 8.6 points; MCID of 7 points), the domain most likely to be affected by an intervention used only during activity. The majority of patients $(n=51(67 \%))$ in this study chose to continue using AOT at study end. Younger age, increased disease severity and improved dyspnoea on patients' global assessment were associated with the decision for continued use of AOT. In the AmbOx trial, the effects of ambulatory oxygen were evaluated over a period of 2 weeks only. Future studies are needed to assess the longer-term effects of oxygen on patient quality of life, activity levels, health resource utilisation and physiological parameters.

Most of the aforementioned studies on AOT were conducted using compressed oxygen cylinders. In recent years, the performance of selected portable oxygen concentrators has been shown to compare favourably with that of compressed oxygen cylinders in in-laboratory assessment studies of patients with COPD and ILD [71, 72]. Compressed oxygen cylinders were the least favoured delivery device in both studies. It is noteworthy that the maximum oxygen delivery capacity of currently available portable oxygen concentrators and compressed oxygen cylinders is limited. The severe levels of exertional desaturation experienced by patients with ILD may not be completely corrected using either type of delivery system [72].

While AOT may have a role in patients with ILD and isolated exertional hypoxaemia, it is clear that it does not improve clinical outcomes in patients with COPD. From the lessons learned in COPD, data from the single assessment studies in ILD need to be interpreted carefully. Although the AmbOx trial has advanced current knowledge regarding the role of AOT in ILD, long-term effects of AOT remain unknown. The development of novel delivery devices enabling greater AOT adherence may impact on its therapeutic potential, and are needed to meet the efficacy, practicality and safety requirements for ambulatory use. In addition to having high oxygen production capabilities, such devices would need to be safe, self-sustaining, lightweight, ergonomic, unobtrusive and affordable.

\section{Nocturnal oxygen therapy in COPD and ILD}

The effects of nocturnal oxygen therapy in patients with COPD and ILD are unclear. There have been three small randomised controlled trials of nocturnal oxygen therapy in COPD, with intervention periods from 6 weeks to 3 years [73-75]. The presence of sleep disordered breathing was investigated using either polysomnography or overnight oximetry in these studies. In 76 patients with COPD and coexisting mild-to-moderate resting hypoxaemia as well as nocturnal desaturation, CHAoust et al. [74] found nocturnal oxygen therapy induced no change in survival or requirement for LTOT after 2 years of follow-up. Similarly, studies in patients with isolated nocturnal hypoxaemia also failed to demonstrate any improvement in survival or health status with nocturnal oxygen therapy [73, 75]. The effects of nocturnal oxygen on pulmonary haemodynamic measurements in these studies are conflicting. CHAOUAT et al. [74] reported no impacts on the evolution of pulmonary vascular measurements with the use of nocturnal oxygen therapy. In contrast, FLETCHER et al. [73] found a significant downward trend in pulmonary arterial 
pressure in patients treated with nocturnal oxygen therapy compared with those who received sham treatment. The effects of nocturnal oxygen therapy in patients with ILD are yet to be explored.

\section{Translating evidence into clinical practice}

Based on the evidence from the landmark trials in COPD, current clinical guidelines from different regions have consistently recommended LTOT for both COPD and ILD patients with significant resting hypoxaemia, defined as resting $P_{\mathrm{aO}_{2}} \leqslant 55 \mathrm{mmHg}(7.3 \mathrm{kPa})$ or $56-59 \mathrm{mmHg}(7.4-8.0 \mathrm{kPa})$ with evidence of chronic hypoxaemia (cor pulmonale, polycythaemia and/or pulmonary hypertension) [76-82].

In contrast, recommendations for AOT in patients with COPD and ILD vary substantially across different regions. The British Thoracic Society recommends prescription of AOT only to patients who are on LTOT and who have preserved outdoor mobility [79]. The Thoracic Society of Australia and New Zealand guideline has a strong recommendation for supplementing stationary concentrator use with AOT in patients using LTOT in order to maximise the number of hours spent with hypoxaemia reversed, while acknowledging the level of evidence is very low [80]. For patients who are ineligible for LTOT with isolated exertional hypoxaemia, there is a weak recommendation for prescribing AOT if demonstrable improvements are observed in symptoms or exercise capacity during a blinded assessment with oxygen versus air. In addition to its use in those who demonstrate an improved exercise capacity during exercise testing, the German Society for Pneumology and Respiratory Medicine guideline also recommends AOT in patients with moderate resting hypoxaemia (defined as resting $P_{\mathrm{aO}_{2}} \leqslant 60 \mathrm{mmHg}$ ) who experience significant exertional hypoxaemia (defined as a reduction of $P_{\mathrm{aO}} \geqslant 5 \mathrm{mmHg}$ during ergometric assessment) [82]. Some national societies recommend consideration of AOT in patients with ILD who experience dyspnoea or exercise limitation due to exertional desaturation [76, 81].

Similarly, there are inconsistent guideline recommendations for the use of nocturnal oxygen therapy. The British Thoracic Society recommends against nocturnal oxygen therapy for patients with both COPD and ILD, unless they meet the eligibility criteria for LTOT [79]. In contrast, the Thoracic Society of Australia and New Zealand guideline states that nocturnal oxygen therapy may be considered in patients with chronic lung diseases and nocturnal desaturation (defined as $\mathrm{S}_{\mathrm{pO}_{2}} \leqslant 88 \%$ for more than one-third of the night), particularly in the presence of hypoxia-related sequelae [80].

Heterogeneity in current guideline recommendations for AOT and nocturnal oxygen therapy reflects the lack of high-level evidence in this area. In addition, variation in funding availability and preconceptions of oxygen therapy from both physicians and patients have a significant impact on clinical practice. Current domiciliary oxygen therapy guidelines were written between 2008 and 2016. It is important that the latest evidence from the LOTT and AmbOx trials be incorporated into new guidelines and translated into clinical practice to improve patient care. Despite the consistent recommendation regarding LTOT, adherence to prescribing guidelines is poor, with marked variation across different regions, ranging from $14 \%$ to $63 \%[15,83-86]$. In order to translate evidence into clinical practice, it will be essential for national and international societies to work together with health bureaucracies to update and implement clinical guidelines, provide educational resources and monitor prescriber adherence.

\section{Conclusions}

Despite $>60$ years of the therapeutic application of oxygen, our understanding of the appropriate clinical use of domiciliary oxygen therapy remains limited. It has taken $>30$ years to prove the inefficacy of domiciliary oxygen therapy in patients with COPD and moderate hypoxaemia after the initial confirmed therapeutic benefits in those with severe resting hypoxaemia. The role of supplemental oxygen therapy for patients with COPD and ILD with exertional or nocturnal hypoxaemia only remains uncertain. Despite a paucity of evidence, prescription of supplemental oxygen therapy is common in both COPD and ILD, and is associated with significant healthcare costs. Further studies are currently underway to clarify the therapeutic potentials of various types of domiciliary oxygen therapy in COPD and ILD (ClinicalTrials.gov identifiers: NCT01044628, NCT02551068, NCT03441204 and NCT03737409; Australian New Zealand Clinical Trials Registry identifier: ACTRN12617000054314). Given that this is an evolving area, timely updates of clinical guidelines and prescribing criteria for domiciliary oxygen therapy are crucial to incorporate new evidence into practice for optimal patient outcomes.

Conflict of interest: Y.H. Khor reports grants from Boehringer Ingelheim, personal fees from Boehringer Ingelheim and Roche, and in-kind support for a clinical trial from Air Liquide Healthcare, outside the submitted work. E.A. Renzoni reports lecture fees and advisory board fees from Boehringer Ingelheim and from Roche, lecture fees from Mundipharma, outside the submitted work. D. Visca has nothing to disclose. C.F. McDonald reports grants from Boehringer Ingelheim, in-kind support for a clinical trial from Air Liquide Healthcare, and speaker's fees paid to her hospital by Menarini and AstraZeneca, outside the submitted work. N.S.L. Goh reports personal fees from Roche and 
AstraZeneca, grants and personal fees from Boehringer Ingelheim, and in-kind support for a clinical trial from Air Liquide Healthcare, outside the submitted work.

\section{References}

1 Ward JJ. History of the respiratory care profession. In: Hess DR, MacIntyre NR, Mishoe SC, et al., eds. Respiratory Care: Principles and Practice. 2nd Edn. Sudbury, Jones \& Bartlett, 2011; pp. 1143-1169.

2 Cotes J, Gilson J. Effect of oxygen on exercise ability in chronic respiratory insufficiency; use of a portable apparatus. Lancet 1956; 9: 872-876.

3 Barach A. Ambulatory oxygen therapy: oxygen inhalation at home and out-of-doors. Dis Chest 1959; 35: 229-241.

4 Ekström M, Ahamadi Z, Larsson H, et al. A nationwide structure for valid long-term oxygen therapy: 29-year prospective data in Sweden. Int J Chron Obstruct Pulmon Dis 2017; 12: 3159-3169.

5 Serginson JG, Yang IA, Armstrong JG, et al. Variability in the rate of prescription and cost of domiciliary oxygen therapy in Australia. Med J Aust 2009; 191: 549-553.

6 Dunne PJ. The demographics and economics of long-term oxygen therapy. Respir Care 2000; 45: 223-228.

$7 \quad$ Nishi SP, Zhang W, Kuo YF, et al. Oxygen therapy use in older adults with chronic obstructive pulmonary disease. PLoS One 2015; 10: e0120684.

8 Phillips M, Cataneo RN, Greenberg J, et al. Effect of oxygen on breath markers of oxidative stress. Eur Respir J 2003; 21: 48-51.

9 Carpagnano GE, Kharitonov SA, Foschino-Barbaro MP, et al. Supplementary oxygen in healthy subjects and those with COPD increases oxidative stress and airway inflammation. Thorax 2004; 59: 1016-1019.

10 Foschino Barbaro MP, Serviddio G, Resta O, et al. Oxygen therapy at low flow causes oxidative stress in chronic obstructive pulmonary disease: prevention by $N$-acetyl cysteine. Free Radic Res 2005; 39: 1111-1118.

11 Stulce JM, Biddle C, Vacchiano C. Low-flow domiciliary oxygen as a mechanism of ongoing oxidative stress Respir Care 2019; in press [https://doi.org/10.4187/respcare.05618].

12 Khor YH, Goh NSL, McDonald CF, et al. Oxygen therapy for interstitial lung disease. A mismatch between patient expectations and experiences. Ann Am Thorac Soc 2017; 14: 888-895.

13 Kelly CA, Maden M. How do respiratory patients perceive oxygen therapy? A critical interpretative synthesis of the literature. Chron Respir Dis 2014; 11: 209-228.

14 Landers A, Wiseman R, Pitama S, et al. Patient perceptions of severe COPD and transitions towards death: a qualitative study identifying milestones and developing key opportunities. NPJ Prim Care Respir Med 2015; 25 15043.

15 Morrison D, Skwarski K, MacNee W. Review of the prescription of domiciliary long term oxygen therapy in Scotland. Thorax 1995; 50: 1103-1105.

16 Kampelmacher MJ, van Kesteren RG, Alsbach GPJ, et al. Characteristics and complaints of patients prescribed long-term oxygen therapy in the Netherlands. Respir Med 1998; 92: 70-75.

17 Neri M, Melani AS, Miorelli AM, et al. Long-term oxygen therapy in chronic respiratory failure: a Multicenter Italian Study on Oxygen Therapy Adherence (MISOTA). Respir Med 2006; 100: 795-806.

18 Sundh J, Ekström M. Risk factors for developing hypoxic respiratory failure in COPD. Int J Chron Obstruct Pulmon Dis 2017; 12: 2095-2100.

19 Wells JM, Estepar RSJ, McDonald MN, et al. Clinical, physiologic, and radiographic factors contributing to development of hypoxemia in moderate to severe COPD: a cohort study. BMC Pulm Med 2016; 16: 169.

20 Noble PW, Albera C, Bradford WZ, et al. Pirfenidone in patients with idiopathic pulmonary fibrosis (CAPACITY): two randomised trials. Lancet 2011; 377: 1760-1769.

21 King TE Jr, Bradford WZ, Castro-Bernardini S, et al. A phase 3 trial of pirfenidone in patients with idiopathic pulmonary fibrosis. N Engl J Med 2014; 370: 2083-2092.

22 Khor YH, Goh NSL, Glaspole I, et al. Exertional desaturation and prescription of ambulatory oxygen therapy in interstitial lung disease. Respir Care 2019; 64: 299-306.

23 Lama VN, Flaherty KR, Toews GB, et al. Prognostic value of desaturation during a 6-minute walk test in idiopathic interstitial pneumonia. Am J Respir Crit Care Med 2003; 168: 1084-1090.

24 Lettieri CJ, Nathan SD, Browning RF, et al. The distance-saturation product predicts mortality in idiopathic pulmonary fibrosis. Respir Med 2006; 100: 1734-1741.

25 Stolz D, Boersma W, Blasi F, et al. Exertional hypoxemia in stable COPD is common and predicted by circulating proadrenomedullin. Chest 2014; 146: 328-338.

26 Andrianopoulos V, Franssen FME, Peeters JPI, et al. Exercise-induced oxygen desaturation in COPD patients without resting hypoxemia. Respir Physiol Neurobiol 2014; 190: 40-46.

27 Villalba WO, Sampaio-Barros PD, Pereira MC, et al. Six-minute walk test for the evaluation of pulmonary disease severity in scleroderma patients. Chest 2007; 131: 217-222.

28 Jenkins S, Čečins N. Six-minute walk test: observed adverse events and oxygen desaturation in a large cohort of patients with chronic lung disease. Intern Med J 2011; 41: 416-422.

29 Delourme J, Stervinou-Wemeau L, Salleron J, et al. Six-minute stepper test to assess effort intolerance in interstitial lung diseases. Sarcoidosis Vasc Diffuse Lung Dis 2012; 29: 107-112.

30 Crisafulli E, Iattoni A, Venturelli E, et al. Predicting walking-induced oxygen desaturations in COPD patients: a statistical model. Respir Care 2013; 58: 1495-1503.

31 García-Talavera I, Figueira-Gonçalves JM, Gurbani N, et al. Clinical characteristics of COPD patients with early-onset desaturation in the 6-minute walk test. Pulmonology 2018; 24: 275-279.

32 Du Plessis JP, Fernandes S, Jamal R, et al. Exertional hypoxemia is more severe in fibrotic interstitial lung disease than in COPD. Respirology 2018; 23: 392-398.

33 Kim C, Seo JB, Lee SM, et al. Exertional desaturation as a predictor of rapid lung function decline in COPD. Respiration 2013; 86: 109-116.

34 Flaherty KR, Andrei AC, Murray S, et al. Idiopathic pulmonary fibrosis: prognostic value of changes in physiology and six-minute-walk test. Am J Respir Crit Care Med 2006; 174: 803-809.

35 Vainshelboim B, Kramer MR, Izhakian S, et al. Physical activity and exertional desaturation are associated with mortality in idiopathic pulmonary fibrosis. J Clin Med 2016; 5: E73. 


\section{Ameer F, Carson} disease who are not hypoxaemic at rest. Cochrane Database Syst Rev 2014; 6: CD000238.

65 Moore RP, Berlowitz DJ, Denehy L, et al. A randomised trial of domiciliary, ambulatory oxygen in patients with COPD and dyspnoea but without resting hypoxaemia. Thorax 2011; 66: 32-37.

66 Vergeret J, Brambilla C, Mounier L. Portable oxygen therapy: use and benefit in hypoxaemic COPD patients on long-term oxygen therapy. Eur Respir J 1989; 2: 20-25.

67 Lacasse Y, Lecours R, Pelletier C, et al. Randomised trial of ambulatory oxygen in oxygen-dependent COPD. Eur Respir J 2005; 25: 1032-1038.

68 Casaburi R, Porszasz J, Hecht A, et al. Influence of lightweight ambulatory oxygen on oxygen use and activity patterns of COPD patients receiving long-term oxygen therapy. COPD 2012; 9: 3-11.

69 Visca D, Mori L, Tsipouri V, et al. Effect of ambulatory oxygen on quality of life for patients with fibrotic lung disease (AmbOx): a prospective, open-label, mixed-method, crossover randomised controlled trial. Lancet Respir Med 2018; 6: 759-770.

70 Sinha A, Patel AS, Siegert RJ, et al. The King's Brief Interstitial Lung Disease (KBILD) questionnaire: an updated minimal clinically important difference. BMJ Open Respir Res 2019; 6: e000363.

71 Strickland SL, Hogan MT, Hogan RG, et al. A randomized multi-arm repeated-measures prospective study of several modalities of portable oxygen delivery during assessment of functional exercise capacity. Respir Care 2009; 54: $344-349$. 

interstitial lung disease: a randomized crossover trial. Respirology 2017; 22: 1598-1603. sleep desaturation in patients with chronic obstructive pulmonary disease and a daytime $\mathrm{Pa}_{\mathrm{O}_{2}}$ above $60 \mathrm{~mm} \mathrm{Hg}$. Am Rev Respir Dis 1992; 145: 1070-1076.

74 Chaouat A, Weitzenblum E, Kessler R, et al. A randomized trial of nocturnal oxygen therapy in chronic obstructive pulmonary disease patients. Eur Respir J 1999; 14: 1002-1008.

75 Orth M, Walther JW, Yalzin S, et al. Influence of nocturnal oxygen therapy on quality of life in patients with COPD and isolated sleep-related hypoxemia: a prospective, placebo-controlled cross-over trial. Pneumologie 2008 62: $11-16$.

76 Bradley B, Branley HM, Egan JJ, et al. Interstitial lung disease guideline: the British Thoracic Society in collaboration with the Thoracic Society of Australia and New Zealand and the Irish Thoracic Society. Thorax 2008; 63: Suppl. 5, v1-v58.

77 Raghu G, Collard HR, Egan JJ, et al. An official ATS/ERS/JRS/ALAT statement: idiopathic pulmonary fibrosis: evidence-based guidelines for diagnosis and management. Am J Respir Crit Care Med 2011; 183: 788-824.

78 Cottin V, Crestani B, Valeyre D, et al. Diagnosis and management of idiopathic pulmonary fibrosis: French practical guidelines. Eur Respir Rev 2014; 23: 193-214.

79 Hardinge M, Annandale J, Bourne S, et al. British Thoracic Society guidelines for home oxygen use in adults. Thorax 2015; 70: Suppl. 1, i1-i43.

80 McDonald CF, Whyte K, Jenkins S, et al. Clinical Practice Guideline on Adult Domiciliary Oxygen Therapy: Executive summary from the Thoracic Society of Australia and New Zealand. Respirology 2016; $21: 76-78$.

81 Funke-Chambour M, Azzola A, Adler D, et al. Idiopathic pulmonary fibrosis in Switzerland: diagnosis and treatment. Respiration 2017; 93: 363-378.

82 Magnet FS, Schwarz SB, Callegari J, et al. Long-term oxygen therapy: comparison of the German and British Guidelines. Respiration 2017; 93: 253-263.

83 Heaney LG, Buick JB, Lowry RC, et al. Prescription of oxygen concentrators and survival in Northern Ireland. Ulster Med J 1997; 66: 86-91.

84 Veale D, Chailleux E, Taytard A, et al. Characteristics and survival of patients prescribed long-term oxygen therapy outside prescription guidelines. Eur Respir J 1998; 12: 780-784.

85 Ringbaek TJ, Lange P, Viskum K. Geographic variation in long-term oxygen therapy in Denmark: factors related to adherence to guidelines for long-term oxygen therapy. Chest 2001; 119: 1711-1716.

86 Verduri A, Ballerin L, Simoni M, et al. Poor adherence to guidelines for long-term oxygen therapy (LTOT) in two Italian university hospitals. Intern Emerg Med 2014; 9: 319-324. 